\title{
Philosophiques
}

\section{Lautman et la réalité des mathématiques}

\section{David Corfield}

Volume 37, numéro 1, printemps 2010

Albert Lautman, philosophe des mathématiques

URI : https://id.erudit.org/iderudit/039714ar

DOI : https://doi.org/10.7202/039714ar

Aller au sommaire du numéro

\section{Éditeur(s)}

Société de philosophie du Québec

\section{ISSN}

0316-2923 (imprimé)

1492-1391 (numérique)

Découvrir la revue

\section{Citer cet article}

Corfield, D. (2010). Lautman et la réalité des mathématiques. Philosophiques, 37(1), 95-109. https://doi.org/10.7202/039714ar

\section{Résumé de l'article}

Cet article examine la thèse de Lautman selon laquelle la réalité des mathématiques doit être approchée par la "réalisation des idées dialectiques ". Pour ce faire, nous reprenons deux exemples que Lautman a lui-même traités. La question est de savoir si on peut ou non mieux décrire les idées dialectiques comme mathématiques, particulièrement maintenant que les moyens mathématiques d'approcher ces idées au niveau de généralisation appropriée existent. Ainsi, la théorie des catégories, inconnue de Lautman, peut donner une description très approfondie de l'idée de dualité. Je soumets de plus que les instances, données par Lautman, de la réalisation des idées dialectiques en dehors des mathématiques et de la physique mathématique sont assez maigres, ce qui suggère fortement que les idées qu'il a décrites si admirablement sont immanentes à la pratique des mathématiques, au lieu d'appartenir à « une réalité idéale, supérieure aux mathématiques ". 


\title{
Lautman et la réalité des mathématiques
}

\author{
DAVID CORFIELD \\ University of Kent \\ D.Corfield@kent.ac.uk
}

\begin{abstract}
RÉSUMÉ. - Cet article examine la thèse de Lautman selon laquelle la réalité des mathématiques doit être approchée par la «réalisation des idées dialectiques ». Pour ce faire, nous reprenons deux exemples que Lautman a lui-même traités. La question est de savoir si on peut ou non mieux décrire les idées dialectiques comme mathématiques, particulièrement maintenant que les moyens mathématiques d'approcher ces idées au niveau de généralisation appropriée existent. Ainsi, la théorie des catégories, inconnue de Lautman, peut donner une description très approfondie de l'idée de dualité. Je soumets de plus que les instances, données par Lautman, de la réalisation des idées dialectiques en dehors des mathématiques et de la physique mathématique sont assez maigres, ce qui suggère fortement que les idées qu'il a décrites si admirablement sont immanentes à la pratique des mathématiques, au lieu d'appartenir à «une réalité idéale, supérieure aux mathématiques».
\end{abstract}

\begin{abstract}
This paper examines Lautman's claim that the reality of mathematics is to be addressed through the "realisation of dialectical ideas". This is done in the context of two examples treated by Lautman himself. The question is raised as to whether we might better describe dialectical ideas as mathematical ones, especially now that we have mathematical means to approach these ideas at the right level of generality. For example, category theory, unknown to Lautman, can describe the idea of duality very thoroughly. It is argued that the instances given by Lautman of the realisation of dialectical ideas outside of mathematics and mathematical physics are rather slight, leading us to conclude that the ideas he so brilliantly describes are immanent to mathematical practice, rather than belonging to "an ideal reality, superior to mathematics".
\end{abstract}

\section{Lautman et la réalité des mathématiques}

C'est avec un grand plaisir que j'ai accepté l'invitation à collaborer à ce volume. Il y a plusieurs années, alors que j'étudiais à Paris en vue d'une carrière possible en psychanalyse, j'en vint à m'intéresser à la philosophie des mathématiques, ce qui n'a rien d'étonnant si l'on se rappelle la façon dont la théorie psychanalytique en France met à contribution les entreprises intellectuelles les plus diverses. Bref, le nom d'Albert Lautman fut prononcé, et je dénichai à la librairie du Centre Georges Pompidou un livre de lui, une édition de ses œuvres publiée en 1977 chez 10/18. À l'époque, une grande partie du cadre philosophique de son travail m'échappa, mais je fus fasciné par les exemples variés de similarité de structures que j'y trouvai et qui me rappelèrent ce que j'avais le plus apprécié au cours de mes études de premier cycle en mathématiques.Au même moment, inspiré par la lecture de Uses and Abuses of the History of Topos Theory de Colin McLarty (1990), je me 
familiarisai avec la théorie des catégories, en particulier grâce à l'ouvrage de Lambek and Scott, Introduction to Higher-Order Categorical Logic, et il ne fut pas difficile d'en inférer que si Lautman avait survécu à la guerre, il aurait adopté sans réserve la théorie des catégories. Peu après, je retournai à Londres pour poursuivre des études en vue de l'obtention d'une maîtrise et d'un doctorat en philosophie des mathématiques, et c'est seulement alors que je commençai à mesurer le gouffre séparant le traitement des mathématiques par Lautman de l'approche philosophique analytique dominante qui prévalait aux États-Unis et au Royaume-Uni. Alors que Lautman pouvait parler des connections de Galois et des fonctions thêta, de la dualité de Poincaré et de la théorie des corps de classes, celles-ci demeuraient des sujets de discussion pour la philosophie anglophone aussi probables que la saison des moussons dans le sud de l'Inde, ou le cycle reproducteur du petit rhinolophe. La meilleure occasion que je puisse imaginer de m'occuper de mathématiques véritables me fut fournie grâce à l'invitation de Lakatos d'appliquer la structure de son programme de recherche, conçue pour la science, aux mathématiques. Tout en tenant compte de ses exigences, les sujets étudiés pouvaient être choisis à volonté dans les parties des mathématiques jugées les plus attrayantes. La structure quelque peu rigide de Lakatos, qui instaurait une rivalité complète entre des programmes de recherche parallèles, rendait cependant très difficile de traiter du sujet favori de Lautman, à savoir l'imbrication des théories dans les mathématiques du $\mathrm{xx}^{\mathrm{e}}$ siècle.

Voici donc une occasion de retourner à Lautman et aux origines des thèses que je soutiens depuis vingt ans:

1. Plutôt que d'accorder à la logique la priorité philosophique sur les autres parties des mathématiques, nous devrions la considérer comme n'importe quelle autre branche, c'est-à-dire comme un lieu où des concepts clés se manifestent de manière récurrente.

2. La réalité des mathématiques doit être abordée par le biais de ces manifestations récurrentes, la «réalisation des idées dialectiques» comme le formule Lautman, et non par le biais des notions quiniennes d'engagement ontologique.

[2] Je désire examiner dans le présent article la deuxième de ces thèses dans le contexte de quelques exemples fournis par Lautman, et soulever certaines questions concernant la nature mathématique de ses «idées dialectiques».

\section{La montée vers l'absolu'}

S'il y a un chapitre dont je me souviens bien vingt ans plus tard, c'est de $L a$ montée vers l'absolu de Lautman. Ici nous trouvons, conjuguées d'une

1. En français dans le texte [NDLT]. 
manière brillante, les manifestations dans la philosophie et dans les mathématiques d'une idée concernant l'imperfection et la perfection. Cette idée en deux parties est celle dans laquelle il est affirmé que tout cas d'imperfection présuppose une perfection correspondante, et qu'il est possible de comprendre les attributs de la perfection à travers les défauts de l'entité imparfaite qui lui est associée. La réalisation de cette idée se manifeste en philosophie dans l'argument de Descartes selon lequel nous pouvons connaître l'existence d'un être parfait, ainsi que ses attributs, en prenant conscience de nos propres imperfections. Par exemple, nous doutons parfois au lieu de savoir, ce qui constitue une imperfection, par conséquent nous pouvons affirmer qu'un être parfait est omniscient.

Lautman consacre davantage de temps à des exemples mathématiques tirés de la théorie algébrique des nombres et de la topologie algébrique. $\mathbb{Q}$ est imparfait, parce qu'il ne permet pas d'effectuer la division du polynôme $x^{2}-2$. Il manque à $\mathbb{Q}$ un élément qui corresponde à la racine carré de 2 , quelque chose qu'un $\mathbb{Q}$ parfait doit par conséquent comporter. Le corps $\mathbb{Q}(\sqrt{2})$ est un pas vers la perfection, mais nous pouvons faire également la même démonstration pour les autres polynômes, par conséquent le $\mathbb{Q}$ parfait doit se confondre en fait avec sa propre fermeture algébrique. Tournonsnous à présent vers la topologie: le cercle est imparfait, parce qu'un lacet peut ne pas y être contractile à un point. Un lacet dessinant un seul circuit autour d'un cercle, par exemple, ne peut pas être contracté à un point. D'un autre côté, le lacet occupant le même circuit dans un cercle faisant deux fois le tour du cercle original est contractile. Cela dit, bien que ce cercle doublé comporte moins d'imperfection, il demeure que tout chemin dans celui-ci formé d'une boucle dans le cercle original et enroulé un nombre pair de tours non égal à zéro ne sera pas contractile. Dans ce cas, le cercle parfait est une hélice. Ces deux exemples tirés des mathématiques ont beaucoup en commun: un treillis de corps intermédiaires, d'une part, et un treillis de revêtements intermédiaires, d'autre part, peuvent y être associés à certains treillis de sous-groupes.

Comme l'a observé Jean Dieudonné dans sa préface à l'édition 10/18, en choisissant ces exemples, Lautman a fait preuve d'une grande sensibilité concernant le type de similarité de structures qui prévaut dans les mathématiques contemporaines, et dont le langage de la théorie des catégories rend compte extrêmement bien. En effet, l'introduction par Grothendieck de la notion de foncteur fibre constitue un moment très important dans l'unification des constructions de la topologie et de la théorie des nombres. Grâce à cette notion, les mathématiciens peuvent s'efforcer de comprendre en théorie des nombres les manifestations de la non-commutativité du groupe fondamental. Rappelons que dans le cas topologique, le groupe fondamental est le groupe de lacets basés à déformation près. En topologie proprement dite, nous pouvons construire une description galoisienne des autres imperfections représentées par des groupes d'homotopie supérieurs. 
Cette veine extrêmement riche des mathématiques est assurément loin d'être épuisée. De fait, une version remise à jour du chapitre de Lautman couvrirait, comme chez Yves André (2008), les équations différentielles, les motifs, les périodes et la renormalisation. Nous disposons cependant, à la différence de Lautman, de la notion selon laquelle le cœur de sa théorie, son «idée », peut être formulée [3] dans un langage mathématique, du moins dans la mesure où l'idée se manifeste en mathématiques. Les mathématiques peuvent aborder l'idée à un niveau d'abstraction au-dessus de ses manifestations. De fait, lorsque Saunders Mac Lane, dans son article intitulé «The Protean Character of Mathematics» (Mac Lane 1992), se sert de l'idée galoisienne pour fournir des preuves à l'appui de sa thèse selon laquelle «la même structure mathématique est susceptible d'un grand nombre de réalisations empiriques différentes»(p. 3), il conclut sa liste de contributions majeures avec:

Janelidze, 1988. Formulation catégorique de la structure de Galois

Divers, 1990. Une addition rend compte de Galois et de bien plus (p. 13),

Or une question, dès lors, se pose: quel rôle le terme «dialectique » remplit-il ? Pourquoi ces idées primitives qui se manifestent si clairement en mathématiques ne sont-elles pas simplement «mathématiques»? Au temps où aucun langage n'existait qui puisse rendre compte des traits communs entre les extensions des corps galoisiens et les transformations de revêtement de Poincaré, il pouvait paraître plausible de considérer ceux-ci comme quelque chose au-delà des mathématiques. Mais cela fait-il sens aujourd'hui ?

Peut-être la solution réside-t-elle dans le rappel que l'idée du parfait et de l'imparfait ne se manifeste pas seulement en mathématiques. Souvenonsnous que l'exemple fourni par Lautman d'une réalisation non mathématique était l'argument de Descartes en faveur de l'existence de Dieu résultant de la conscience de ses propres imperfections. Nous pourrions soutenir qu'une partie de ce qui se manifeste ici n'est pas mathématique, et que par conséquent l'idée commune tant à cet exemple philosophique qu'à l'exemple galoisien n'est elle-même pas mathématique. Certes, il pourrait sembler que la mathématisation de l'argument de Descartes n'offre pas un grand intérêt. Mais nous pourrions nous demander s'il n'est pas significatif que la manifestation en philosophie soit plutôt, disons, mince, alors que les manifestations en mathématiques sont énormément plus riches. Qui plus est, si nous entendons capturer l'essence de l'exemple mathématique, la question se pose de savoir si la perfection et l'imperfection sont les meilleurs termes à utiliser. Galois lui-même semble avoir été davantage enclin à penser en termes d' "ambiguité » plutôt que d'imperfection. Dans sa dernière lettre il écrit:

Mes principales méditations, depuis quelque temps, étaient dirigées sur l'application de la théorie de l'ambiguïté à l'analyse transcendante. 
Il s'agissait de voir a priori, dans une relation entre des quantités ou fonctions transcendantes, quels échanges on pouvait faire, quelles quantités on pouvait substituer aux quantités données, sans que la relation pût cesser d'avoir lieu. Cela fait reconnaître tout de suite l'impossibilité de beaucoup d'expressions que l'on pourrait chercher.

Mais je n'ai pas le temps, et mes idées ne sont pas encore bien développées sur ce terrain, qui est immense (passage cité dans André 2008).

Bien entendu, l'ambiguité peut être considérée comme une forme d'imperfection, mais à moins que le cas cartésien ne soit compris comme une forme d'ambiguité, les traits communs entre celui-ci et le cas galoisien nous paraîtront assurément bien affaiblis.

Par ailleurs, bien que Lautman évite le terme, la description des universaux par Michael Polanyi en tant que «signification commune de choses formant une classe» est pertinente ici. Cette signification, soutient-il, est "quelque chose de réel puisqu'elle est capable de se manifester indéfiniment dans le futur». Il poursuit:

Elle possède en effet un pouvoir heuristique qui est habituellement double. (1) Un concept universel anticipe ordinairement l'occurrence d'instances supplémentaires de lui-même dans le futur, et si le concept est vrai, il [4] subsumera correctement ces futures instances en dépit du fait qu'elles différeront de manière imprévisible dans chaque particulier de toutes les instances subsumées dans le passé. (2) Un véritable concept universel, désignant une classe naturelle, par exemple une espèce d'animaux, anticipe chez les membres de la classe en question la découverte d'une gamme indéfinie de propriétés non conventionnelles; c'est-à-dire la découverte que cette classe possède une portée intensionnelle encore inconnue (Polanyi 1969, 170-171).

Pour donner un exemple de ces pouvoirs, lorsqu'il a été suggéré que les souris et les éléphants appartenaient à une même classe, appelée «mammifères ", nous aurions pu nous attendre à ce que (1) des animaux très différents s'avèrent être des mammifères, et (2) qu'une gamme indéfinie de traits communs entre les souris et les éléphants soit découverte. Dans ce cas, si l'on considère la subsomption des dauphins aquatiques dépourvus de jambes sous la classe, et la découverte d'importantes similarités physiologiques et, ultimement, génétiques entre les souris et les éléphants, ces pouvoirs ont été admirablement mis en œuvre.

Je pense que nous pouvons courir ici le risque de négliger les différences entre les universaux et les idées, et nous demander si les traits communs qui se manifestent dans les conceptions respectives de Descartes et de Galois possèdent de semblables pouvoirs heuristiques. De la même façon que dans (1), nous pouvons chercher ailleurs une autre manifestation riche de la dialectique de la perfection et de l'imperfection. Nous pouvons certainement retrouver la théorie de Galois employée en physique, chez Gepner 2006 par exemple, mais il n'est pas évident que la dialectique de la perfection et de 
l'imperfection soit pertinente pour ces travaux en théorie des champs conformes rationnelle. Ailleurs, la théorie de l'homotopie rend compte des défauts dans les cristaux nématiques liquides (Nash et Sen 1983, chap. 9). Cependant, et bien que la connotation d'imperfection soit présente, il n'est pas évident que de l'existence d'un cristal défectueux nous arrivions à la notion correspondante d'un cristal parfait et sans défaut. En outre, si l'on considère ce cas comme une troisième manifestation de l'idée dialectique de Lautman, il nous faut noter la relation étroite qu'il entretient avec les mathématiques. Il ne peut que difficilement constituer un troisième point dans un triangle formé par les manifestations de l'idée dialectique, se confondant plutôt, quasiment, avec un point de mathématiques.

D'un autre côté, de la même façon que dans (2), peut-être que l'apparente minceur de l'exemple philosophique est illusoire. Peut-être pourrait-il se révéler être plus galoisien que nous ne le pensions. Il y a bien une manière évidente, me semble-t-il, d'enrichir cet exemple, une manière qui nous est fortement suggérée par nos plus récentes connaissances mathématiques. Si l'idée galoisienne était apparue dans son plein développement à Descartes, celui-ci se serait vu forcé de combiner toute la hiérarchie des différentes substructures composant le complexe des imperfections humaines et celle des différentes sortes d'êtres angéliques s'interposant entre l'Homme et Dieu. Thomas d'Aquin a décrit, paraît-il, dans sa Summa Theologica une hiérarchie d'anges disposés en trois rangs de trois niveaux chacun. Je ne serais pas du tout surpris que cette théorie angélologique médiévale, ou une autre tout aussi élaborée, puisse être reformulée sous une forme peu ou prou galoisienne.

Mais peut-être que l'exemple mathématique était de toute façon quelque peu spécial. Il est certain qu'une théorie mathématique ne rend pas compte dans tous les cas des divers exemples de réalisation commune en mathématiques aussi bien que dans ce cas-ci. Considérons un autre exemple présenté par Lautman.

\section{La réciprocité et la dualité}

Dans les «Nouvelles recherches sur la structure dialectique des mathématiques» (1939), Lautman traite de l'usage de l'analyse dans la théorie des nombres. Il remarque que [5] cet usage a mis certains mal à l'aise et qu'ils ont cherché à l'éliminer. Mais Lautman ne voit nulle nécessité métaphysique à cette «purification ». Au lieu de concevoir l'arithmétique comme métaphysiquement antérieure à l'analyse, il propose plutôt que nous les considérions à un degré égal comme des réalisations des mêmes structures dialectiques.

Il donne comme exemple celui des entités réciproques. En arithmétique, nous avons la réciprocité quadratique, dans laquelle les symboles de Legendre fonctionnent en quelque sorte comme des inverses les uns des autres.

$$
\left(\frac{p}{q}\right)\left(\frac{q}{p}\right)=(-1)^{\frac{(p-1)}{2} \frac{(q-1)}{2}}
$$


Ici, pour les nombres premiers impairs $p$ et $q$, le premier symbole de Legendre prend la valeur +1 , si p est un carré modulo q; autrement, il prend la valeur -1 .

Lautman remarque en outre qu'il a été possible de généraliser la réciprocité de deux manières différentes. Premièrement, aux entiers algébriques dans tout corps. Deuxièmement, pour permettre des congruences plus générales, non seulement au carré, mais à d'autres puissances. Cela a été accompli algébriquement, note-t-il, mais il s'empresse d'ajouter que Hecke a également fourni les méthodes analytiques de dériver des résultats généraux de réciprocité quadratique à l'aide des fonctions thêta.

Nous définissons ici:

$$
\theta(\tau)=\sum_{m=-\infty}^{m=+\infty} e^{-\pi \tau m^{2}}
$$

en notant que les points singuliers sont à $r=2 i r$ ( $r$ étant un nombre rationnel), mais que pour tout $r, \sqrt{ } r \theta(r+2 i r)$ prend une valeur finie qui est, "à quelques facteurs sans importance près, la somme de Gauss $\mathrm{C}(-r)$ » (Lautman 2006, p. 248). Nous avons par ailleurs la propriété transformationnelle de la fonction thêta:

$$
\theta(1 / \tau)=\sqrt{\tau \theta(\tau)}
$$

et cela nous dit qu'il existe une relation réciproque entre $\mathrm{C}(r)$ et $\mathrm{C}(-1 / 4 r)$, de laquelle s'ensuit la réciprocité quadratique ordinaire. Lautman affirme:

Cette idée dialectique de réciprocité entre éléments inverses peut être si nettement distinguée de ses réalisations en arithmétique ou en analyse, qu'il est possible de trouver un certain nombre d'autres théories mathématiques en lesquelles elle se réalise pareillement (Lautman 2006, p. 248).

Notons toutefois, avant d'examiner les autres cas de réalisations de réciprocité repérés par Lautman, qu'il est important pour celui-ci que l'idée dialectique soit clairement distinguée de ses réalisations. Nous pouvons nous demander une fois de plus ce qui maintient les mathématiques des années 30 dans l'incapacité de capturer, ou du moins d'aborder l'idée de réciprocité elle-même. L'obtention d'un cadre qui puisse rendre compte de l'ensemble des réalisations a dû paraître à l'époque une perspective lointaine, d'autant plus que Lautman invoque également certains travaux très récents d'André Weil indiquant l'existence d'un lien entre les lois de réciprocité et la dualité de Poincaré. Cette dualité relie l'homologie de dimension $m$ et la cohomologie de dimension $n-m$ dans une variété de dimension $n$, ce qui dans certains cas de variétés implique une relation simple entre homologies de dimensions complémentaires. Lautman a encore une fois brillamment choisi son exemple. Il existe effectivement des traits communs entre la réciprocité quadratique [6] et la dualité de Poincaré, lesquels traits entretiennent un lien 
étroit avec l'idée galoisienne de la dernière section. J'esquisserai brièvement ce point dans ce qui suit.

Bien après Lautman, dans les années 70, Barry Mazur et David Mumford ont remarqué une puissante analogie entre les idéaux dans les corps de nombres algébriques et les liens dans les variétés de dimension 3, se présentant comme suit: dans la perspective de la cohomologie étale, les nombres entiers relatifs $\mathbb{Z}$ ou plutôt le schéma $\operatorname{Spec}(\mathbb{Z})$ a l'apparence d'une sorte de sphère à trois dimensions. Un nombre premier $p$ a un schéma associé $\mathrm{K}_{p}=\operatorname{Spec}(\mathbb{Z} / p \mathbb{Z})$ qui dans la même perspective a l'apparence d'une sous-variété de dimension 1 de cette sphère. Un grand progrès peut maintenant être réalisé en appliquant les idées de la théorie des nœuds à la théorie algébrique des nombres. La dualité de Poincaré peut alors être utilisée pour définir l'enlacement entre deux «nœuds » $\mathrm{K}_{p}$ et $\mathrm{K}_{q}$. La réciprocité quadratique, dans ce cadre, ne signifie pas autre chose que le fait que l'enlacement entre les nœuds $A$ et $B$ est le même que l'enlacement entre les nœuds $B$ et $A$ (Waldspurger 1976).

La partie analytique de la question a aussi été énormément développée depuis l'époque de Lautman, permettant entre bien autres choses de faire le lien entre la réciprocité des fonctions L et la dualité de Poincaré. Nous touchons ici aux domaines les plus brûlants en mathématiques contemporaines, nommément le monumental programme de Langlands et les motifs de Grothendieck. Le sens de l'unité des mathématiques était chez Lautman extrêmement aiguisé. Il possédait une capacité phénoménale à nous orienter vers la réalité mathématique. Cependant, avec le succès à indiquer la réalité mathématique vient en même temps la question de déterminer ce qui n'est pas mathématique dans les idées qui se manifestent ici. Bien que nous n'en soyons pas encore au point où nous disposerions d'une théorie mathématique compréhensive de toutes les formes de dualité, il existe du moins un fort sentiment selon lequel l'idée de dualité elle-même, et non pas ses manifestations spécifiques, peut être abordée mathématiquement. Ici encore, le langage de la théorie des catégories, ainsi que celui de sa cousine la théorie des catégories de dimensions supérieures, est requis, les dualités étant souvent exprimables en termes d'équivalences.

Dans le chapitre 7 de leur ouvrage Sets for Mathematics (2003), Lawvere et Rosebrugh introduisent une distinction utile entre la dualité «formelle» et la dualité "concrète». La dualité formelle concerne les simples inversions de flèches dans un diagramme résultant, par rapport à un diagramme original, de telle sorte que:

bien entendu, si les diagrammes originaux se sont vus conférer une interprétation spécifique en termes d'ensembles et d'applications spécifiques, une telle interprétation est perdue lorsque nous passons à cette duale formelle, parce que le processus de dualisation formelle en lui-même ne détermine pas des ensembles et des applications spécifiques qui permettent d'interpréter l'énoncé dualisé (p. 121). 
La dualité concrète, d'un autre côté, apparaît dans des situations où un nouveau diagramme est formé à partir d'un ancien en exponentiant chaque objet relativement à un objet dualisant donné, par exemple, $X$ devient $V^{x}, V$ étant l'objet dualisant. Les flèches sont naturellement inversées dans le nouveau diagramme. Cependant,

tout énoncé ne sera pas converti en sa duale formelle par le processus de dualisation relativement à $V$, et de fait une grande partie de l'étude des mathématiques espace versus quantité et de la logique théorie versus exemple

[7] peut être considérée comme l'étude détaillée du degré auquel la dualité formelle et la dualité concrète dans un $V$ déterminé correspondent ou échouent à correspondre (p. 122).

Or, contrairement au cas de la dialectique de la perfection et de l'imperfection, Lautman ne fait aucune allusion à une quelconque réalisation non mathématique de l'idée de la réciprocité et de la dualité, mais si le programme de Langlands doit se voir conférer une importance philosophique pour le reste de la philosophie en raison du fait qu'il constitue un cas de manifestation d'une idée qui se manifeste également dans d'autres sphères de la vie, il nous faudra trouver quelques exemples intéressants. Nous pourrions faire certaines suggestions, mais le problème serait assurément que celles-ci pâliraient jusqu'à l'insignifiance en comparaison de l'exemple mathématique. Notre meilleure chance serait de nous tourner à nouveau vers la physique, et de considérer par exemple la dualité entre le champ électrique et le champ magnétique. Cependant, encore une fois, les exemples physiques ont énormément en commun avec les exemples mathématiques. Les uns comme les autres partagent en effet toute une histoire entrecroisée, faite d'influence et de développement mutuels, depuis la théorie de Hodge et la cohomologie de de Rham, jusqu'à la symétrie miroir, la S-dualité et la T-dualité de la théorie des cordes. Kapustin et Witten (2006) mettent en rapport la dualité électro-magnétique avec une partie du programme de Langlands. D'après de nombreuses indications, la théorie des catégories jouerait, encore une fois, un rôle clé.

Mais peut-être que le problème de Lautman est qu'il nous oriente instinctivement vers les manifestations d'une même idée en mathématiques qui est elle-même, ultimement, abordable par la théorie mathématique. Certaines suggestions donnent à penser que ce n'est pas le cas de la totalité des mathématiques.

\section{Les deux cultures des mathématiques}

Alors que le sens esthétique de Lautman relatif aux mathématiques l'a poussé à choisir de préférence des exemples qui se sont ultimement révélés être abordables par la théorie des catégories, un tel sens esthétique se manifeste couramment sous d'autres formes. Ainsi, bien que je sois persuadé que Lautman aurait beaucoup apprécié les sujets de discussion sur le blogue que 
je co-dirige, The n-Category Café, le blogue écrit par Terence Tao révèle une sensibilité assez différente. L'essai de Timothy Gowers intitulé The Two Cultures of Mathematics constitue selon moi la meilleure tentative de rendre compte de cette différence. L'auteur introduit à cet effet dans cet ouvrage la distinction entre «constructeurs de théories» et « résolveurs de problèmes». Je suis d'avis que nous devons nous montrer très prudents avec ces étiquettes, comme l'est Gowers lui-même:

$[\ldots]$ quand je dis que les mathématiciens peuvent être classés en deux types: les constructeurs de théories et les résolveurs de problèmes, je parle de leurs priorités, plutôt que de faire l'affirmation ridicule selon laquelle ils seraient exclusivement voués à une seule sorte d'activité mathématique (p. 2).

Afin d'éviter les malentendus, peut-être est-il par conséquent préférable de donner tout de suite des exemples paradigmatiques de travaux issus de chacune de ces cultures.

- Constructeurs de théories: géométrie algébrique de Grothendieck, programme de Langlands, symétrie miroir, cohomologie elliptique.

- Résolveurs de problèmes: théorie combinatoire des graphes, théorème de Ramsey, théorème de Szemerédi, progressions arithmétiques dans les nombres premiers.

[8] Gowers mentionne Sir Michael Atiyah comme l'exemple par excellence d'un constructeur de théories, et recommande la lecture de ses essais informels, les "General Papers ", publiés dans le premier volume de ses Collected Works. Ceux-ci expriment en effet une esthétique pour laquelle j'en suis venu à éprouver une énorme admiration au cours de mon doctorat en philosophie. D'un autre côté, Paul Erdös est mentionné comme un résolveur de problèmes consommé. Qu'en est-il donc de l'esthétique correspondante?

L'un des attraits des sujets de prédilection des résolveurs de problèmes, que Gowers réunit sous la dénomination générale de "combinatoire ", est l'accessibilité facile des problèmes.

L'une des grandes satisfactions des mathématiques est que, juchés sur des épaules de géants, comme le dit le dicton, nous pouvons atteindre à des hauteurs insoupçonnées des générations antérieures. Toutefois, la plupart des articles en combinatoire sont indépendants, ou exigent tout au plus un petit nombre de connaissances d'arrière-plan de la part du lecteur. Comparons cela avec un théorème en théorie algébrique des nombres, dont la compréhension peut prendre des années pour un débutant muni des connaissances du niveau d'un programme de premier cycle typique (p. 12).

Pour quelqu'un récemment décoré de la médaille Fields, il pourrait sembler étrange d'éprouver le besoin de justifier ses propres intérêts, mais après avoir décrit un problème concernant les nombres de Ramsey, Gowers écrit : 
Je considère ce problème comme l'un des problèmes principaux en combinatoire et j'ai consacré de nombreux mois de ma vie à tenter de le résoudre sans succès. Et pourtant je me sens presque embarrassé d'écrire cela, conscient que je suis que plusieurs mathématiciens regarderaient la question davantage comme un jeu de casse-tête que comme un problème mathématique sérieux (p. 11).

Il est fréquent que l'on fasse appel, comme garants de l'importance de son propre domaine, aux liens que celui-ci entretient avec d'autres domaines et à son applicabilité. Or,

[e]n ce qui concerne les liens avec d'autres sujets, il existe des applications de la combinatoire à la probabilité, à la théorie des ensembles, à la cryptographie, à la théorie de la communication, à la géométrie des espaces de Banach, l'analyse harmonique, la théorie des nombres... la liste est encore longue. Toutefois, je suis conscient en écrivant cela qu'un bon nombre de ces applications ne parviendraient pas à impressionner un géomètre différentiel, par exemple, qui pourrait les considérer toutes comme appartenant d'une manière ou d'une autre à cette partie en quelque sorte étrangère des mathématiques qu'on peut ignorer sans dommage. Même les applications à la théorie des nombres le sont à la «mauvaise sorte» de théorie des nombres (p. 13).

Le théorème de Green-Tao, concernant les longueurs des progressions arithmétiques dans les nombres premiers, peut paraître un bon candidat pour illustrer cette "mauvaise sorte» de théorie des nombres. De fait, celuici a fourni à Minhyong Kim une belle manière de représenter la différence entre cultures. Laquelle des descriptions suivantes nous séduit le plus?

- Le théorème concernant les nombres premiers dans les progressions arithmétiques.

- Le théorème concernant les progressions arithmétiques dans les nombres premiers.

[9] La première d'entre elles est celle d'un résultat de Dirichlet, correspondant à une étape précoce dans la théorie algébrique des nombres (un des sujets préférés des constructeurs de théories).

Certes, ce n'est pas que, dans le camp des constructeurs de théories, tous les résultats théoriques concernant les nombres entiers issus de la "bonne sorte» de théorie des nombres soient jugés importants. Il est indéniable que l'on a que trop usé et abusé du théorème de Fermat au fil des années. C'est plutôt l'activité féconde à l'arrière-scène, laquelle aura mené à la preuve de la conjecture de Taniyama-Shimura, qui est considérée en général comme l'accomplissement majeur. Par conséquent, même si l'on estimait que les résultats concernant l'existence de progressions arithmétiques dans les nombres premiers étaient pareillement galvaudés, un résultat général pourrait, cette fois-ci encore, se dissimuler à l'arrière-scène. Selon Gowers, cependant, on a affaire en combinatoire non pas tant à des théorèmes qu'à des principes généraux. Par exemple, 
[...] si l'on tente de maximiser la taille d'une structure quelconque sous certaines contraintes, et si les contraintes semblent forcer les exemples extrémaux à s'étaler de manière uniforme, alors choisir au hasard un exemple a de bonnes chances de mener à une bonne réponse (p. 6).

Le fait que la théorie des catégories ne joue aucun rôle dans la «combinatoire » de Gowers n'est pas un accident. Par conséquent, si l'on accorde de l'importance à l'activité mathématique selon ses impacts sur les mathématiques dans leur ensemble, alors en lieu et place de l'application des résultats théoriques et des outils d'un domaine à un autre (et que la théorie des catégories a grandement facilitée), il peut s'avérer nécessaire de considérer des relations plus subtiles, telles que:

[quand] le domaine A est suffisamment proche dans son esprit du domaine B, [...] quiconque est bon en A est susceptible d'être bon en B. Qui plus est, de nombreux mathématiciens contribuent aux deux domaines (p. 14).

Il semble bien, pourtant, que l' «esprit » commun puisse être exprimé sous forme d'idée. Par exemple, dans son article, The dichotomy between structure and randomness, arithmetic progressions, and the primes (Tao 2005), Terence Tao nous parle des manifestations de l'idée de la structure et du hasard dans une bonne partie de ses travaux. Ce terrain s'annonce-t-il plus prometteur pour Lautman?

Remarquons premièrement que l'état des lieux actuel en mathématiques ne devrait pas nous induire en erreur. Il est possible qu'une théorie générale plus explicite émergera qui saura rendre compte des résultats de Tao et Gowers, tout comme une théorie abstraite de l'idée dialectique galoisienne a émergé depuis l'époque de Lautman. Pour des considérations sur une possible réconciliation en ce qui concerne les deux approches de la théorie des nombres, voir Kim 2007. Cependant, même si aucune théorie explicite de ce genre ne rend compte du couple de la structure et du hasard, comme la formulation en termes de théorie des catégories le fait de l'idée galoisienne, il me semble que nous ne pouvons faire autrement que de considérer l'article de Tao comme mathématique. S'il n'y a pas d'autre accès à une idée excepté un accès mathématique, je ne vois pas pourquoi l'idée en question ne devrait pas être vue comme mathématique.

\section{Conclusion}

L'étude lautmanienne de la réciprocité prend place immédiatement après une présentation des idées de Heidegger sur la différence entre «ontologique» et " ontique», "essence» et "existence». Je n'ai fait nulle mention de cette discussion ici, trouvant ce langage quelque peu étranger [10] au mien. Je me suis plutôt attardé sur les tentatives que Lautman fait pour situer la source de la réalité des mathématiques en un lieu qui se trouve au-delà des mathématiques. 
Nous voudrions montrer, avant de conclure, comment cette conception d'une réalité idéale, supérieure aux mathématiques et pourtant si prête à s'incarner dans leur mouvement, vient s'intégrer dans les interprétations les plus autorisées du platonisme (Lautman 2006, p. 230)².

J'ai soutenu que cette "réalité idéale ${ }^{3}$ ne se situe pas au-delà des mathématiques mais constitue plutôt le cœur des mathématiques ellesmêmes, et que dans de nombreux cas, elle ne peut être abordée mathématiquement à un niveau plus abstrait que par le biais de ses instantiations dans des théories mathématiques spécifiques.

Une position différente voudrait considérer le traitement des idées à ce niveau comme philosophique. William Lawvere, dans son article intitulé «Categories of Space and of Quantity» (Lawvere 1992), écrit:

C’est ma conviction que dans la prochaine décennie et dans le prochain siècle les avancées techniques réalisées par les théoriciens des catégories se révéleront valables pour la philosophie dialectique, conférant une forme précise avec des modèles mathématiques susceptibles d'examen critique à d'anciennes distinctions philosophiques telles que le général et le particulier, l'objectif et le subjectif, l'être et le devenir, l'espace et la quantité, l'égalité et la différence, le quantitatif et le qualitatif, etc. En retour, l'attention explicite de la part des mathématiciens à de telles questions philosophiques est nécessaire pour réussir à rendre les mathématiques (et donc les autres sciences) davantage assimilables et utilisables. Bien entendu, cela exigera que les philosophes apprennent les mathématiques et que les mathématiciens apprennent la philosophie (p. 16).

Mais la manière dont nous désignons le genre de travaux commun à Lautman et à Lawvere est en réalité de peu d'importance. Ce qui est important, d'un autre côté, est que nous reconnaissions le rôle central que ces travaux devraient jouer dans la compréhension philosophique des mathématiques. Même si j'ai raison de vouloir appeler mathématiques l'extraction de l'idée galoisienne et d'autres idées semblables, Lautman nous a quand même rendu un énorme service en se révélant un guide aussi fiable vers la réalité mathématique. Si l'on souhaite réfléchir sur cette réalité, on ne peut mieux faire que d'en examiner les développements théoriques présentés en partie dans les exemples qu'il étudie. Par exemple, les idées véhiculées dans le programme de Langlands sont des composantes essentielles de la réalité mathématique. Mais plutôt que de nous orienter vers une réalité supérieure aux mathématiques, j'estime qu'il est préférable pour nous de considérer cet exemple comme l'illustration d'un cas où une discipline traite de sa propre réalité.

L'intérêt des mathématiques pour la philosophie, par conséquent, est qu'elles fournissent à cette dernière un exemple supplémentaire de la notion de réalité.

2 En français dans le texte (texte corrigé) (NDLT)

3 En français dans le texte (NDLT) 
Cette notion se manifeste dans les mathématiques d'une manière, dans la biologie d'une autre, dans la politique d'une autre et dans l'art d'une autre encore. Le talent de Lautman a été de faire preuve extraordinairement tôt d'une sensibilité esthétique appelée à dominer de larges sections des mathématiques du vingtième siècle. Cette sensibilité n'est rien de moins que le plus puissant mode d'accès que les êtres humains aient pu avoir à la réalité mathématique jusqu'à présent. Grâce à elle, l'esprit est devenu plus conforme à son objet.

Un autre philosophe qui considérait nos efforts pour étendre notre savoir et le mouvement de nos théories commes des indices du réel était Michael Polyanyi. Sa conception de la réalité est «ce qui peut encore et toujours se manifester inexhaustiblement»(Polyanyi 1969, p. 141). Au sujet des mathématiques, il écrit: [11]

[...] tandis que dans les sciences naturelles le sentiment de prise de contact avec la réalité est un augure de confirmations empiriques futures et non encore imaginées d'une découverte immanente, en mathématiques il présage une gamme indéterminée de germinations futures à l'intérieur des mathématiques elles-mêmes (Polyanyi 1958, p. 189).

Ce qui précède est une description de la réalité mathématique qui me paraît pleinement satisfaisante. Chez certains, notamment chez Galois, Poincaré et Grothendieck, ce sentiment se sera avéré juste, si l'on en juge par la gamme extraordinairement riche de germinations futures résultant de leurs œuvres.

Traduit de l'anglais par Anne-Marie Boisvert

\section{Bibliographie}

André, Y. Ambiguity theory, old and new, 2008 [en ligne:http://arxiv.org/abs/ 0805.2568].

Baez, J. et M. Shulman. Lectures on n-Categories and Cohomology, 2006 [en ligne: http://arxiv.org/abs/math/0608420].

Gepner, D. Galois Groups in Rational Conformal Field Theory, 2006 [en ligne: http :// arxiv.org/abs/hep-th/0606081].

Gowers, W. T. "The Two Cultures of Mathematics ", in Mathematics: Frontiers and Perspectives, V. I. Arnold et al. (dir.), American Mathematical Society, 1999.

Kapustin, A. et E. Witten. Electric-Magnetic Duality and The Geometric Langlands Program, 2006 [en ligne: http://arxiv.org/abs/hep-th/0604151].

Kim,M.Blog comment, 2007 [en ligne :http :/golem.ph.utexas.edu/category/2007/04/ the two cultures of mathematic.html\#c009058].

Lambek, J. et P. J. Scott. Introduction to Higher-Order Categorical Logic, Cambridge University Press.

Lawvere, F. W. "Categories of Space and of Quantity ", in The Space of mathematics, Echeverria et al. (dir.), Walter de Gruyter, 1992. 
Mac Lane, S. «The Protean Character of Mathematics » in The Space of mathematics, Echeverria et al. (dir.), Walter de Gruyter, 1992.

McLarty, C. Uses and Abuses of the History of Topos Theory, British Journal for the Philosophy of Science 41(3), 1990, 351-375.

Nash, C. et S. Sen. Topology and Geometry for Physicists, Academic Press, 1983.

Polanyi, M. Personal Knowledge, Routledge, 1958.

- Knowing and Being, University of Chicago Press, 1969.

Tao, T. The dichotomy between structure and randomness, arithmetic progressions, and the primes, 2005 [en ligne: http://arxiv.org/abs/math/0512114].

Waldspurger, J.-L. 1976, Entrelacements sur Spec(Z), Bull. Sci. Math. (2) 100, n 2, 1976, 113-139. 